\title{
Photochemical investigation of a photochromic diarylethene compound that can be used as a wide range actinometer
}

\author{
André Ribeiro Santos, ${ }^{* a}$ Roberto Ballardini, ${ }^{b}$ Peter Belser, ${ }^{c}$ Maria Teresa Gandolfi, ${ }^{b}$ Vijay Mahadevan Iyer ${ }^{d}$ and \\ Luca Moggi $* b$
}

The photochromic diarylethene derivative 1,2-bis(5-(4-ethynylphenyl)-2-methylthiophen-

3-yl)perfluorocyclopentene (1) was submitted to photochemical, thermal stability and fatigue resistance studies in acetonitrile, also to evaluate its possible application as a new actinometer. This photochromic system covers a wide spectral absorption range, with intense bands in the UV and visible regions for the open-ring and closed-ring isomers, respectively. Very high ring-closure quantum yield values were obtained, in contrast with the low ring-opening quantum yields, which are nevertheless high enough to exploit $\mathbf{1}$ as an actinometer. The procedure required to determine the photon flux of an irradiation source with this fatigue resistant compound is indeed very simple.

\section{Introduction}

"Photochromism is the reversible transformation of a chemical species between two forms having different absorption spectra, induced in one or both directions by absorption of electromagnetic radiation". 1,2

Diarylethenes are typical photochromic molecules in which the thermodynamically stable colorless open-ring isomer is converted to the colored closed-ring isomer upon irradiation (see, for example, Scheme 1), while the reverse reaction is either thermally or photochemically activated. ${ }^{3,4}$

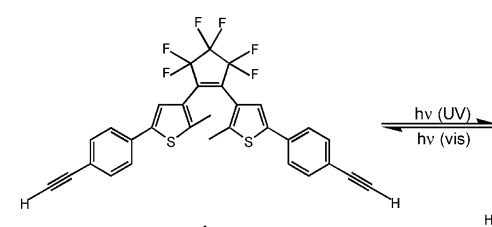

$1 a$

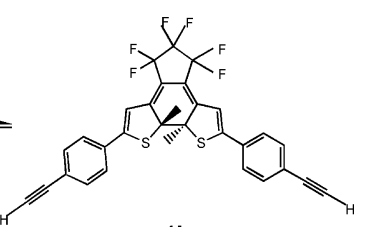

$1 b$
Scheme 1 Open-ring (1a) and closed-ring (1b) isomers of 1,2-bis(5(4-ethynylphenyl)-2-methylthiophen-3-yl)perfluorocyclopentene (1).

The possibility of a thermal reaction occurring in a photochromic system represents a disadvantage when such a system is employed for optical memory or actinometric purposes. Fortunately, many diarylethenes containing heterocyclic rings have been found to exhibit high thermal stability of both isomers, ${ }^{3,4}$ so that both the ring-closure and the reverse ring-opening reactions can only be accomplished photochemically.

$\mathrm{NMR}^{5,6}$ and absorption spectroscopy ${ }^{6}$ studies have shown that five-membered heterocyclic diarylethenes exhibit in solution at

${ }^{a}$ Organic Chemistry Department, Complutense University, Madrid, Spain

b"G. Ciamician" Department of Chemistry, University of Bologna, Via Selmi 2, 40126, Bologna, Italy

'Department of Chemistry, University of Fribourg, Chemin du Musée 9, 1700, Fribourg, Switzerland

${ }^{d}$ Office of Technology Transfer, Temple University, 1601 North Broad Street, 406 University Services Building (083-45), Philadelphia, PA 19122, USA least two open-ring conformations (Scheme 2); the antiparallel conformer presents $C_{2}$ symmetry while the parallel one presents a mirror $C_{\mathrm{s}}$ symmetry.
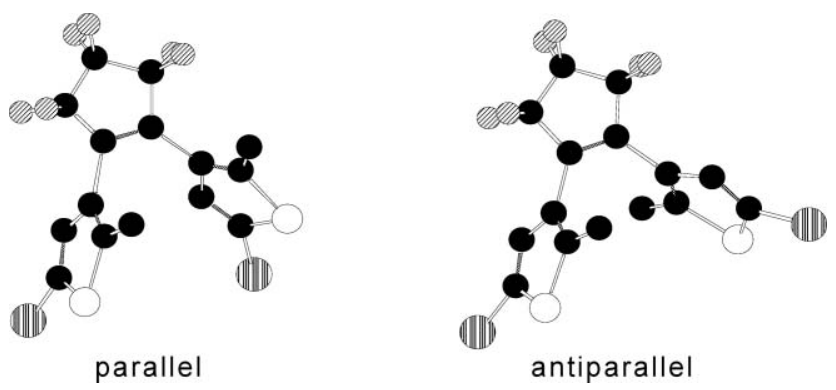

Scheme 2 Schematic representation of parallel and antiparallel conformers of 1,2-bis(2-methylthiophen-3-yl)perfluorocyclopentenes.

According to the Woodward-Hoffmann rule, ${ }^{7}$ the cyclization reaction occurs photochemically in a conrotatory mode, while the corresponding thermal reaction would require a disrotatory mode $;$ therefore, the conrotatory antarafacial photocyclization reaction can only proceed from the antiparallel open-ring conformer. Since the interconversion between the conformers occurs in a time scale larger than the excited-state lifetime, ${ }^{5}$ only the light absorbed by the antiparallel form can induce photocyclization.

The photochromic diarylethene derivative 1,2-bis(5-(4ethynylphenyl)-2-methylthiophen-3-yl)perfluorocyclopentene (1), shown in Scheme 1, has useful spectroscopic properties, since it covers a wide spectral range, and is also expected to be thermally stable in both isomeric forms. ${ }^{4}$ We have therefore studied the thermal and photochemical behaviour as well as the fatigue resistance properties of this system; moreover, considering that its absorption spectrum extends in the visible up to about $700 \mathrm{~nm}$ and that photochromic actinometers require very simple practical procedures, we have evaluated its application as a new chemical actinometer. 


\section{Fatigue resistance}

A photochemical cyclization reaction implies the rearrangement of chemical bonds and it may be accompanied by side or secondary photoreactions leading to irreversible products. When a photochromic system is used in a repetitive way (as occurs in many applications), side reactions cause a progressive chemical degradation of the system and thus a loss of performance over time; this effect is commonly called "fatigue of the system". ${ }^{2}$ The fatigue resistance can be quantified by the number of complete photocoloring/photobleaching cycles necessary to cause a decrease of $20 \%$ in the absorbance of the open-ring or closed-ring isomer; ${ }^{3,4}$ this percentage is more suitable for our purposes than the $50 \%$ percentage quoted as Z50 in ref. 2 .

\section{Experimental section}

\section{Materials}

Compound $\mathbf{1}$ had already been synthesized by one of the authors (Vijay M. Iyer), according to a slight modification of a published method, ${ }^{9}$ and characterized by X-ray studies. ${ }^{10}$

For the synthesis of the 1,2-bis(5-(4-trimethylsilylethynylphenyl)-2-methylthiophen-3-yl)perfluorocyclopentene intermediate (see Scheme 4 of ref. 9), a solution of $n$-BuLi (1.6 $\mathrm{M}$ in hexane, $1.0 \mathrm{~mL}, 1.71 \mathrm{mmol}$ ) at $-78{ }^{\circ} \mathrm{C}$ under argon atmosphere was added to a stirred solution of 3-bromo-2methyl-5-(4-trimethylsilylethynylphenyl)-thiophene $(0.560 \mathrm{mg}$, $1.60 \mathrm{mmol})$ in freshly distilled tetrahydrofuran $(20 \mathrm{~mL})$. After $15 \mathrm{~min}$, perfluorocyclopentene $(0.1 \mathrm{~mL}, 0.8 \mathrm{mmol})$ was added with a cooled syringe as quickly as possible. The reaction mixture was stirred during $2 \mathrm{~h}$ and then was allowed to warm to room temperature. The mixture was quenched with hydrochloric acid $0.1 \mathrm{M}(1.5 \mathrm{~mL})$. The solvents were removed in vacuo and the resulting dark green residue was extracted with diethyl ether. The combined organic extracts were dried with magnesium sulfate, filtered and evaporated. Column chromatography over silica gel with hexane containing $2 \%$ dichloromethane provided a bluish-white solid (248 mg, 43\%).

To obtain 1 , this intermediate $(180 \mathrm{mg}, 0.25 \mathrm{mmol})$ was added to a solution of $\mathrm{NaOH}(148 \mathrm{mg}, 3.7 \mathrm{mmol})$ in methanol $(10 \mathrm{~mL})$ and the resulting mixture was stirred for $3 \mathrm{~h}$ at room temperature. The solvents were removed in vacuo and the greenish residue was extracted with diethylether. The combined ether extracts were dried with magnesium sulfate, filtered and evaporated. Column chromatography over silica gel with hexane containing $2 \%$ dichloromethane provided a bluish-white solid (108 mg, 75\%). The product was further purified in several batches using plate chromatography (silica gel) with the same eluent (82 mg, 57\%). The sample of $\mathbf{1}$ so obtained is mainly in the form $\mathbf{1 a}$ with a small percentage of $\mathbf{1 b}$.

In the present work the separation of the two isomers was performed on silica TLC plates, using the non-polar mixture hexane-toluene $10: 1$ as the mobile phase, ${ }^{11}$ the retention factors were determined to be 0.36 for $\mathbf{1 b}$ and 0.30 for $\mathbf{1 a}$. The blur of the 1b isomer was isolated by scratching the surface of the silica into a beaker, dissolving it in acetonitrile and filtering by means of a syringe equipped with a PTFE filter.
The solvents used for the photochemical experiments (acetonitrile and dichloromethane) were Merck Uvasol. The compounds used as chemical actinometers were samples normally utilized in the Photochemistry Laboratory in Bologna.

\section{Apparatus}

The absorption spectra were recorded using a Cary 50 UVVis spectrophotometer with the software Cary Win UV Scan Application.

Polychromatic visible light used to obtain solutions of pure 1a was supplied by the halogen lamp of a Leica Pradovit P300 projector with a cut-off $(\lambda>500 \mathrm{~nm})$ filter. Monochromatic light for the studies of the photochemical behavior was obtained from a Helios Italquartz $15 \mathrm{~W}$ low pressure mercury lamp (254 nm), or a Helios Italquartz Polymer 125 medium pressure mercury lamp equipped with a LOT-Oriel interference filter $(313,334,404$, or $549 \mathrm{~nm}$ ), or the projector lamp equipped with an interference filter centred at 589 or $609 \mathrm{~nm}$ (half width 14 and $7 \mathrm{~nm}$, respectively); the 334 interference filter was combined with a cut-off filter, to suppress any contribution from 313 and $365 \mathrm{~nm}$ mercury lines.

\section{Procedures}

All experiments were performed under dim red light, at room temperature (unless otherwise noted), with air equilibrated solutions. However, in fatigue resistance experiments, argon-purged solutions were also used.

Photochemical experiments were performed in spectrophotometric cells (Hellma Suprasil, $1 \mathrm{~cm}$ optical path), and during irradiation the solutions were stirred with a Hellma Cuv-o-Stir Model 333 magnetic stirrer.

The intensity (photon flux ${ }^{2}$ ) of the incident light was determined by means of the most reliable actinometric systems, ${ }^{12,13}$ i.e. the ferrioxalate actinometer ${ }^{14,15}$ for the UV light, the Reinecke's salt actinometer ${ }^{16}$ or Aberchrome $540^{17-19}$ (as a control) for the visible light.

In the case of 589 or $609 \mathrm{~nm}$ irradiation, since the actinometer had uneven absorbance in the allowed wavelength interval of the interference filter and the light source was a continuous lamp, the ratio between light transmitted by the filter and light absorbed by the actinometer (which is that actually measured) was wavelength dependent. Assuming that the emission intensity of the lamp is wavelength independent in the filter range, the overall ratio was calculated by means of eqn (1),

$$
I_{\mathrm{o}}=I_{\mathrm{a}} \frac{\sum_{\lambda} T_{\lambda}^{\mathrm{f}}}{\sum_{\lambda}\left(T_{\lambda}^{\mathrm{f}} \times F_{\lambda}^{\mathrm{a}}\right)}
$$

where (see also Fig. 1):

$I_{\mathrm{o}}$ is the overall intensity of the light transmitted by the filter (einstein $\min ^{-1}$ );

$I_{\mathrm{a}}$ is the overall intensity of the light measured by the actinometer (einstein $\min ^{-1}$ );

$T_{\lambda}^{\mathrm{f}}$ is the mean fraction of light transmitted by the filter in $1 \mathrm{~nm}$ width;

$F_{\lambda}^{\mathrm{a}}$ is the mean fraction of light absorbed by the actinometer in $1 \mathrm{~nm}$ width; 


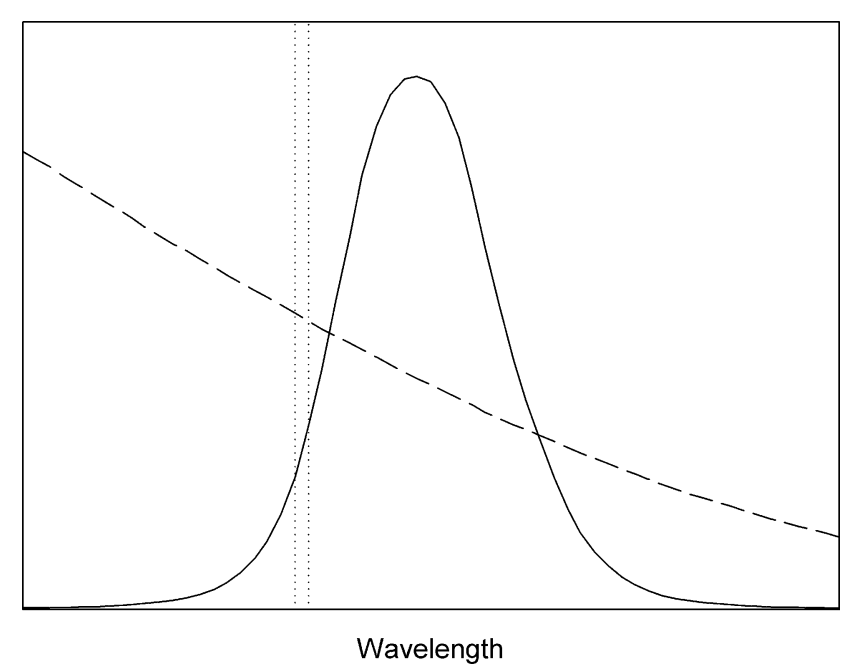

Fig. 1 Schematic representation of the procedure used to calculate the light intensity transmitted by the filter $\left(I_{\mathrm{o}}\right)$ in case of uneven absorption of the actinometer. (-) Filter transmittance; (---) actinometer absorption. For more details, see text.

the summations are extended to the entire allowed wavelength interval.

\section{Ring closure (1a $\rightarrow$ 1b) quantum yield measurement}

$3.0 \mathrm{~mL}$ of a fresh solution of $1\left(\sim 3 \times 10^{-5} \mathrm{~mol} \mathrm{~L}^{-1}\right)$, dissolved in the chosen solvent, were irradiated for a few minutes with visible light $(\lambda>500 \mathrm{~nm})$, until about $100 \%$ of the open-ring isomer 1a was obtained. The solution was then irradiated with UV monochromatic light $(254,313$, or $334 \mathrm{~nm})$ for convenient intervals of time (of the order of a few minutes), with the increase in the absorbance at $600 \mathrm{~nm}$ measured after each interval.

The mathematical expression used to determine the value of the ring-closure quantum yield, $\Phi(\mathbf{1 a} \rightarrow \mathbf{1 b})$, at the irradiation wavelength is given by eqn (2),

$$
\Phi(\mathbf{1} \mathbf{a} \rightarrow \mathbf{1 b})=\frac{\Delta A \times V}{I_{\mathrm{o}} \times\left(1-10^{-A \lambda}\right) \times \varepsilon \times \Delta t}
$$

where:

$\Delta A$ is the increase of absorbance at $600 \mathrm{~nm}$ in the irradiation period $\Delta t$;

$V$ is the volume of irradiated solution $(\mathrm{L})$;

$\varepsilon$ is the molar absorption coefficient of $1 \mathbf{b}$ at $600 \mathrm{~nm}(17400 \mathrm{~L}$ $\mathrm{mol}^{-1} \mathrm{~cm}^{-1}$ );

$I_{\mathrm{o}}$ are the moles of incident photons (einstein $\mathrm{min}^{-1}$ ) determined by the appropriate actinometer;

$A_{\lambda}$ is the absorbance of the solution at the irradiation wavelength (mean value during the irradiation period);

$\Delta t$ is the irradiation time ( $\mathrm{min})$.

\section{Ring-opening $(1 \mathrm{~b} \rightarrow$ 1a) quantum yield measurement}

$3.0 \mathrm{~mL}$ of a fresh solution of $\mathbf{1}\left(\sim 3 \times 10^{-5} \mathrm{~mol} \mathrm{~L}^{-1}\right)$, dissolved in the chosen solvent, were irradiated by a medium pressure mercury lamp with $313 \mathrm{~nm}$ light until almost complete conversion to $\mathbf{1 b}$ was reached. The solution so obtained was then irradiated with visible light $(404,549,589$, or $609 \mathrm{~nm})$ for convenient intervals of time (of the order of a few minutes) and the decrease in the absorbance at $600 \mathrm{~nm}$ measured after each interval.

The mathematical expression used to determine the value of the ring-opening quantum yield, $\Phi(\mathbf{1 b} \rightarrow \mathbf{1 a})$, at the chosen wavelength is again eqn (2), but in this case $\Delta A$ is the decrease in absorbance at $600 \mathrm{~nm}$.

\section{Fatigue resistance evaluation}

Several consecutive complete photocoloring/photobleaching cycles were accomplished on a solution of 1a in the appropriate solvent. Photocoloring (reaction 1a $\rightarrow$ 1b) was performed by irradiating the solution with $313 \mathrm{~nm}$ light, until the absorbance at $600 \mathrm{~nm}$ had reached its maximum constant value (i.e. complete conversion to $\mathbf{1 b}$ ); photobleaching (reaction $\mathbf{1 b} \rightarrow \mathbf{1 a}$ ) by irradiating with visible light $(\lambda>500 \mathrm{~nm})$ until the absorbance at $313 \mathrm{~nm}$ had reached its maximum constant value (i.e. complete conversion to 1a). The progressive consumption of 1 was evaluated by measuring the absorbance at $329 \mathrm{~nm}$ (maximum of 1b) at the end of photocoloring and the absorbance at $313 \mathrm{~nm}$ (maximum of 1a) at the end of photobleaching.

\section{Results}

Preliminary experiments were carried out on solutions of $\mathbf{1}$ in acetonitrile as well as dichloromethane. The results obtained in the latter solvent were not as good as those in the former one with regard to thermal stability, secondary photoproducts, and fatigue resistance properties. Therefore, further extensive investigations were carried out exclusively in acetonitrile solutions.

\section{Characterization of 1 in acetonitrile}

In all the experiments described below the pure isomer 1a was obtained by irradiating solutions of $\mathbf{1}$ with visible polychromatic light $(\lambda>500 \mathrm{~nm})$ for a few minutes, while the isomer $\mathbf{1 b}$ was obtained by irradiating a solution of $\mathbf{1}$ with monochromatic UV light until the spectrum showed constant absorbance in the visible region.

Since the closed forms of diarylethenes absorb ultraviolet light, they could also undergo the reverse ring-opening photoreaction upon UV irradiation and the absorption spectrum so obtained for 1b could not be that of the pure isomer, but that of a photostationary equilibrium state. Actually, the existence of a photostationary state has been reported for numerous compounds; in most cases, however, the amount of the open-ring isomer in such a state is less than $10 \%$ and often it is negligible. ${ }^{4,20-24}$ In order to clarify this point for compound $\mathbf{1}$, preliminary experiments were performed by irradiating the open-ring isomer with light of three different wavelengths, 254, 313, and $334 \mathrm{~nm}$, since different photostationary equilibrium states (and thus, different final spectra) are expected. On the contrary, we found that all the three spectra were very similar in shape and molar absorption coefficients; in addition, they were coincident, within the limits of the experimental uncertainty, with that of pure $\mathbf{1 b}$ obtained by TLC separation. We can thus conclude that the amount of 1a present in the final state, if any, is negligible.

The electronic absorption spectra of both open- and closed-ring isomers are reported in Fig. 2. 1a exhibits only one absorption band with maximum at $313 \mathrm{~nm}\left(\varepsilon=53500 \mathrm{~L} \mathrm{~mol}^{-1} \mathrm{~cm}^{-1}\right)$ with 


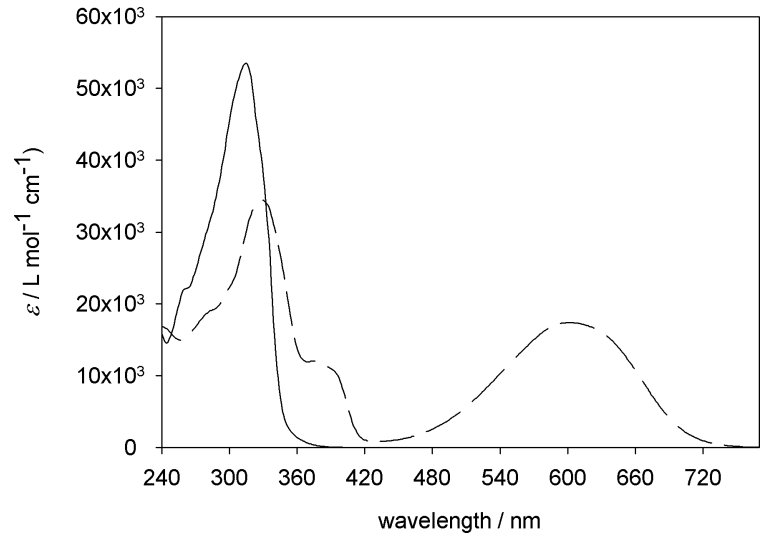

Fig. 2 Absorption spectra of the open-ring (-) and closed-ring (---) isomers of compound $\mathbf{1}$ in acetonitrile.

a shoulder at about $255 \mathrm{~nm}$, and no absorption in the visible. The spectrum of $\mathbf{1 b}$ shows a band with maximum at $329 \mathrm{~nm}$ $\left(\varepsilon=36000 \mathrm{~L} \mathrm{~mol}^{-1} \mathrm{~cm}^{-1}\right)$, a shoulder at $376 \mathrm{~nm}(\varepsilon=11800 \mathrm{~L}$ $\left.\mathrm{mol}^{-1} \mathrm{~cm}^{-1}\right)$, and a second broad band with maximum at $600 \mathrm{~nm}$ $\left(\varepsilon=17400 \mathrm{~L} \mathrm{~mol}^{-1} \mathrm{~cm}^{-1}\right)$. The uncertainty on the $\varepsilon$ values is $\pm 2.5 \%$.

\section{Thermal stability}

The thermal stability of $\mathbf{1}$ has been already reported. ${ }^{9}$ We have found that solutions of $\mathbf{1 b}$ (the thermodynamically unstable isomer) in acetonitrile, stored in the dark at $40{ }^{\circ} \mathrm{C}$, showed no spectral change for at least $950 \mathrm{~min}$, a time period much longer than that required by photochemical experiments performed at room temperature.

\section{Photochemical behaviour}

The photochemical behaviour of the open- and closed-ring isomers was then studied qualitatively and quantitatively by following the changes in the absorption spectra.

Irradiation of solutions of $\mathbf{1 a}$ in acetonitrile with $313 \mathrm{~nm}$ light caused the rapid formation of the broad absorption band in the visible with maximum at $600 \mathrm{~nm}$, characteristic of the closedring isomer 1b (Fig. 3). However, for irradiation periods longer

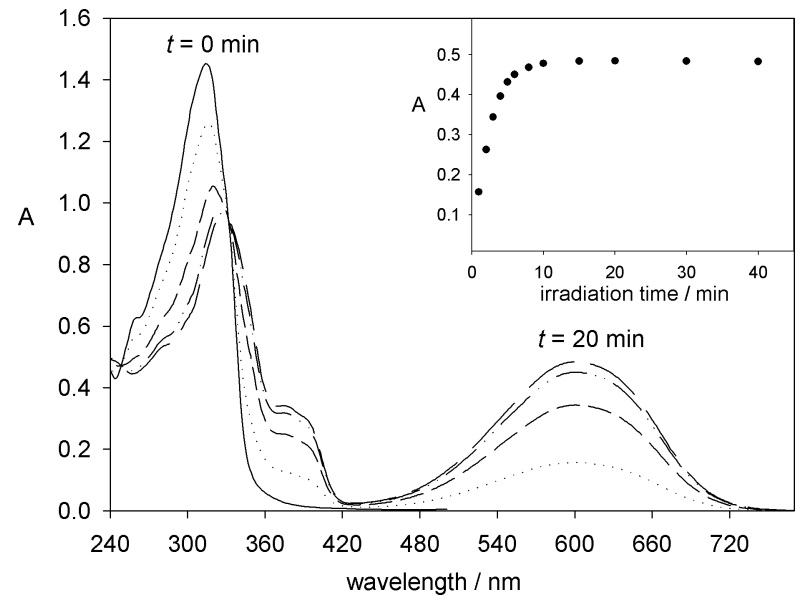

Fig. 3 Conversion of $\mathbf{1 a}$ to $\mathbf{1 b}$ upon irradiation with $313 \mathrm{~nm}$ light in acetonitrile. The reported spectra are those corresponding to $0,1,3,6$ and $20 \mathrm{~min}$ of irradiation. Inset: absorbance at $600 \mathrm{~nm} v s$. irradiation time. than 20 min, a very slow progressive decrease of the absorbance at $600 \mathrm{~nm}$ was observed, due to a secondary photoreaction of 1b (Fig. 3, inset), a behaviour already observed for analogous compounds. $^{24,25}$ Extrapolation to $t=0$ of this linear decrease of absorbance at $600 \mathrm{~nm}$ indicates that an eventual loss of absorbance during the first $20 \mathrm{~min}$ of irradiation (the time required to reach the maximum value of the absorbance) must be smaller than 0.003 : this means that the secondary photoreaction practically may be observed only after the photochemical conversion of $\mathbf{1 a}$ into $\mathbf{1 b}$ is completed; such a conclusion is supported by the presence of two neat isosbestic points in the spectra of the irradiated solution (Fig. 3) and by the fact that the spectrum of $\mathbf{1 b}$ obtained after $20 \mathrm{~min}$ of irradiation is strictly consistent with the spectrum of pure 1b obtained by TLC separation (see above). Similar results were obtained upon 254 or $334 \mathrm{~nm}$ irradiation.

Hence, the general scheme of the photoreactions that occur in a solution of $\mathbf{1}$ may be as shown in Scheme 3.

$$
1 a \frac{h_{v}(U V)}{h v(v i s)}=1 b \stackrel{h v(U V)}{-X}
$$

Scheme 3

The quantum yield values of photoreactions $\mathbf{1 a} \rightarrow \mathbf{1 b}$ and $\mathbf{1 b} \rightarrow \mathbf{1 a}$ are listed in Tables 1 and 2, respectively; as far as reaction $\mathbf{1 b} \rightarrow \mathrm{X}$ is concerned, a quantum yield value of the order of $10^{-3}$ has been estimated for an aerated acetonitrile solution irradiated at $313 \mathrm{~nm}$, from the data of Fig. 3 inset.

\section{Ring closure $(1 \mathrm{a} \rightarrow 1 \mathrm{~b})$ quantum yield}

Experiments on solutions in acetonitrile were carried out using the procedure described above. Fig. 4 shows the quantum yield values calculated by means of eqn (2) for various consecutive irradiation time intervals in a single experiment; their progressive decrease is due to the fact that the photoproduct $\mathbf{1 b}$ absorbs an increasing fraction of the excitation light, while eqn (2) only accounts for the total absorbance of the solution.

The due correction can be easily accomplished by extrapolating the quantum yield values to zero time, where $\mathbf{1 b}$ was practically absent in the solution. ${ }^{26}$ Corrected quantum yield values, as well

Table 1 Corrected ${ }^{a}$ quantum yield values of ring closure for irradiation of $1 \mathbf{a}$ in acetonitrile

\begin{tabular}{lll}
\hline & \multicolumn{1}{c}{$\boldsymbol{}(\mathbf{1} \mathbf{a} \mathbf{1} \mathbf{b})$} & \\
\cline { 2 - 3 }$\lambda / \mathrm{nm}$ & Single experiment ${ }^{b}$ & Average \\
\hline 254 & 0.95 & $0.92 \pm 0.03$ \\
& 0.92 & \\
& 0.89 & \\
313 & 0.67 & $0.71 \pm 0.03$ \\
& 0.70 & \\
& 0.70 & \\
& 0.75 & \\
& 0.69 & \\
& 0.76 & \\
& 0.70 & \\
& 0.72 &
\end{tabular}

${ }^{a}$ Extrapolated to $t=0 .{ }^{b}$ Uncertainty: $\pm 5 \%$. 
Table 2 Quantum yield values of ring opening for irradiation of $\mathbf{1 b}$ in acetonitrile

\begin{tabular}{lll}
\hline & \multicolumn{1}{l}{$\Phi(\mathbf{1 b} \rightarrow \mathbf{1 a})$} & \\
\cline { 2 - 3 }$\lambda / \mathrm{nm}$ & Single experiment ${ }^{a}$ & Average \\
\hline 404 & 0.016 & $0.016 \pm 0.001$ \\
& 0.016 & \\
& 0.016 & \\
& 0.015 & $0.0064 \pm 0.0003$ \\
549 & 0.0063 & \\
& 0.0067 & $0.0032 \pm 0.0002$ \\
589 & 0.0062 & \\
& 0.0034 & $0.0015 \pm 0.0001$ \\
& 0.0030 & \\
609 & 0.0031 & \\
& 0.0013 & \\
& 0.0016 & \\
${ }^{a}$ Uncertainty: $\pm 5 \%$. & & \\
\hline
\end{tabular}

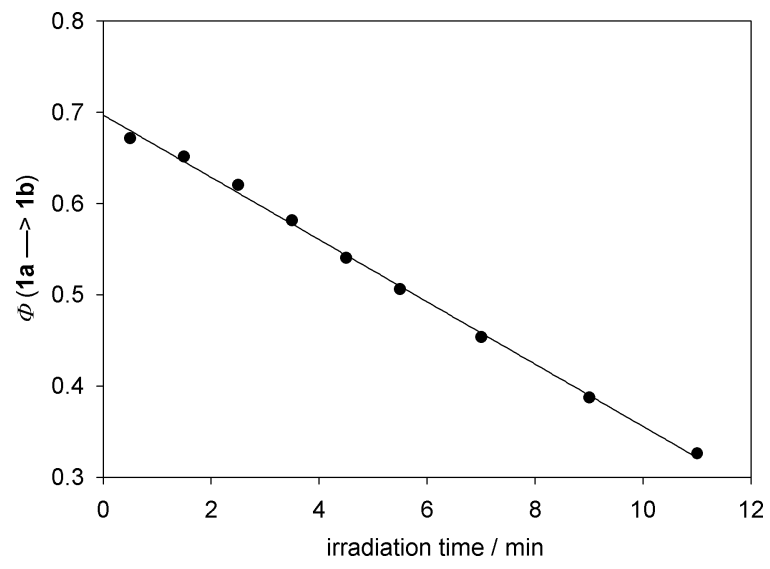

Fig. 4 Plot of the ring closure quantum yield values for consecutive irradiation time intervals $\left(\lambda_{\text {irr }}=313 \mathrm{~nm}\right.$, in acetonitrile).

as their average, for excitation with 254,313 or $334 \mathrm{~nm}$ light are summarized in Table 1. They also show a decrease in going from 254 to $313 \mathrm{~nm}$ excitation, while no significant change occurs between 313 and $334 \mathrm{~nm}$.

\section{Ring-opening (1b $\rightarrow$ 1a) quantum yield}

Experiments on solutions in acetonitrile were carried out using the procedure described above. The quantum yield does not show any dependence on irradiation time, in agreement with the fact that the photoproduct 1a does not absorb visible light. Quantum yield values of various experiments, as well as their average, for excitation with 404, 549, 589, or $609 \mathrm{~nm}$ light are summarized in Table 2.

$\Phi(\mathbf{1 b} \rightarrow \mathbf{1 a})$ is much smaller than $\Phi(\mathbf{1 a} \rightarrow \mathbf{1 b})$; it also decreases with increasing wavelength (Fig. 5), as it occurs for other diarylethene compounds. ${ }^{27,28}$ A good linear fitting of the data can be obtained, so that the ring opening quantum yield can be calculated using the following equation, at least in the $400-610 \mathrm{~nm}$ interval:

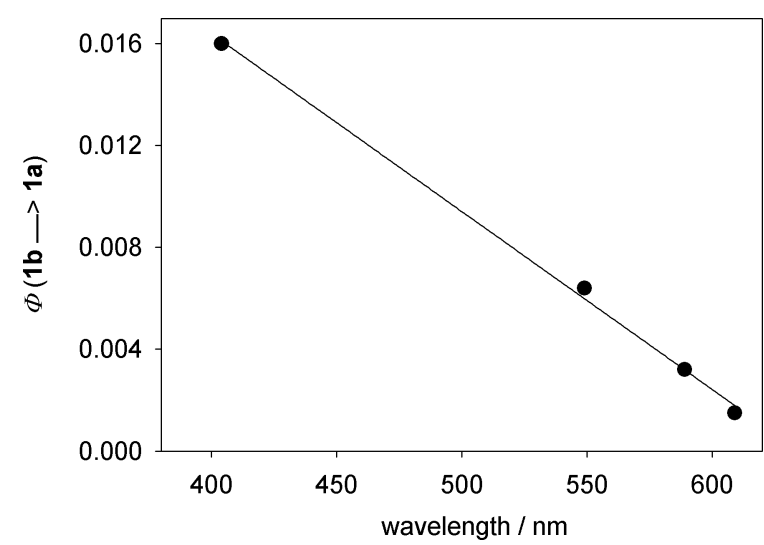

Fig. 5 Ring-opening quantum yield values of $\mathbf{1 b}$ in acetonitrile as a function of the irradiation wavelength.

$$
\Phi(\mathbf{1 b} \rightarrow \mathbf{1 a})=0.0440-6.94 \times 10^{-5} \times \lambda / \mathrm{nm}
$$

\section{Fatigue resistance evaluation}

Fig. 6 reports the results of a fatigue resistance experiment in air-equilibrated solution, with the photocoloring step induced by $313 \mathrm{~nm}$ light. The absorbance of both $\mathbf{1 a}$ and $\mathbf{1 b}$ isomers shows a very small decrease with the increasing number of cycles. A small increase of absorbance appears around $560 \mathrm{~nm}$ at the end of the thirteenth photobleaching step; since it is approximately equal to the decrease of the absorbance in the same spectral region observed before the last bleaching, it should be due to a compound whose absorption coefficients in the region are approximately one half of those of $\mathbf{1 b}$.

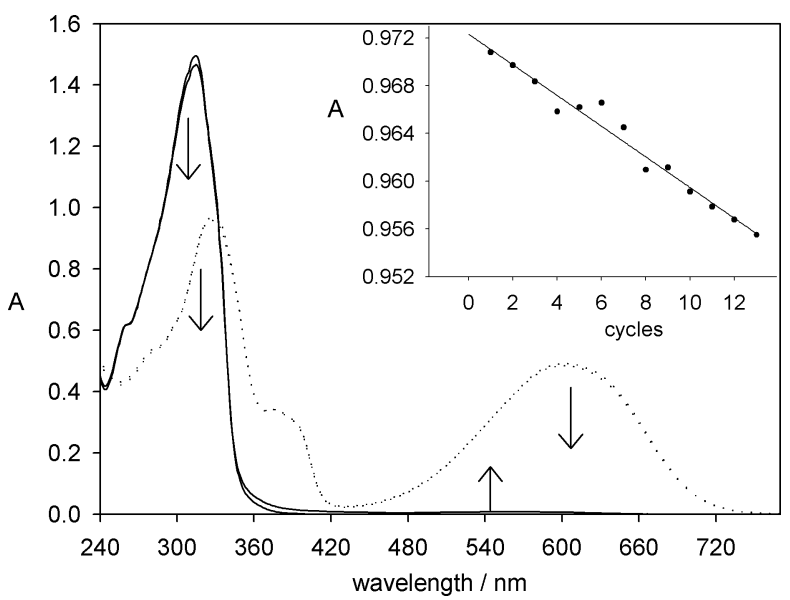

Fig. 6 Spectra of compound $\mathbf{1}$ in aerated acetonitrile solutions in a 13 cycle photocoloring/photobleaching experiment; open-ring form 1a (-); closed-ring form $\mathbf{1 b}$ (---). Inset: values of absorbance at $329 \mathrm{~nm}$ at the end of the photocoloring step of each cycle.

The inset of Fig. 6 shows an example of the progressive decrease of absorbance; from the linear regression of analogous plots we have evaluated the number of cycles required to reduce the absorbance to $80 \%$ of its initial value, summarized in Table 3 . 
Table 3 Fatigue resistance values of 1 in acetonitrile ${ }^{a}$

\begin{tabular}{lll}
\hline & \multicolumn{2}{l}{ Number of cycles } \\
\cline { 2 - 3 } Detection wavelength/nm & Aerated solutions & Deaerated solutions \\
\hline $313^{b}$ & 140 & 190 \\
$329^{c}$ & 150 & 180
\end{tabular}

${ }^{a}$ Photocoloring step: irradiation at $\lambda=313 \mathrm{~nm}$; photobleaching step: irradiation at $\lambda>500 \mathrm{~nm} .{ }^{b}$ Maximum of $\mathbf{1 a} .{ }^{c}$ Maximum of $\mathbf{1 b}$.

\section{Discussion}

\section{Absorption spectra}

The electronic absorption spectra of both isomers of $\mathbf{1}$ are similar to those of most of the compounds of the diarylethene family; the intense absorption bands have been attributed to transitions to singlet excited states, and this attribution has been confirmed by semiempirical calculations. ${ }^{28,29}$ The lowest energy band of most open isomers closely resembles, in shape and position, the first excitation of the free aryl groups, ${ }^{28,30,31}$ and thus can be attributed to transitions within the $\pi$-system of these chromophores. The broad absorption band of the closed isomers in the visible region reflects the large $\pi$-conjugation of the lateral aryl chromophores and the central structure; therefore the red-shifting of these bands correlates with the $\pi$-donating ability of the substituents on the aryl rings. ${ }^{21}$ Among the 1,2-bis(thiophen-3-yl)perfluorocyclopentene derivatives, $\mathbf{1}$ is one of the most "red-shifted" compounds, being overtaken only by derivatives having long polyene chains ${ }^{32}$ or peculiar substituents ${ }^{20,28,29}$ in the 5-position of the thiophene rings, and by compounds containing oligomeric thiophenes. ${ }^{22}$

\section{Thermal stability}

Compound 1 shows high thermal stability for both isomeric forms, therefore the ring-opening reaction of $\mathbf{1 b}$ can only be accomplished photochemically. Such a property is common to almost all the diarylethenes containing furan, thiophene or thiazole rings, while not observed in those containing pyrrole, indole or phenyl rings. ${ }^{3,4,8}$ On the basis of state energy calculations, the thermal stability of closed-ring isomers has been attributed to the large energy barrier of the ring-opening reaction when the ground-state energy difference between open-ring and closed-ring isomers is small; this in turn correlates with the aromatic stabilization energy of aryl groups. ${ }^{8}$ On the other hand, the ground state ring-opening reaction in a disrotatory mode is sterically hindered by the two trans-oriented methyl groups at the 2-position of the thiophene rings. ${ }^{29,33}$

\section{Photochemical behaviour}

The photochemical behaviour of 1, summarized by Scheme 3, is typical of diarylethene compounds. Flash photolysis and transient spectroscopy studies on many of these compounds have evidenced that both the ring-closure and ring-opening photoreactions occur in the picosecond time range, ${ }^{28-31,33-37}$ which means that the reactive excited states are singlet states. As a consequence, the primary photoreactions cannot be affected by the presence of dioxygen in the solution, since a diffusion controlled reaction between the excited state and $\mathrm{O}_{2}$ would a time require about $10^{3}$ longer than the observed lifetimes of the excited states; ${ }^{38}$ actually, no difference was observed between the ring-closure quantum yields in aerated and deaerated solutions of a 1,2-bis(2-methylthiophen3 -yl)perfluorocyclopentene derivative. ${ }^{39}$

Ring-closure photoreaction. The very high ring-closure quantum yield of 1a (see Table 1) is a rather unusual result among the open isomers of diarylethenes: they generally show quantum yield values around 0.5 or lower, at least in non-polar solvents, as a consequence of the almost equal distribution between antiparallel (photochemically reactive) and parallel (unreactive) conformers. ${ }^{5,21-23,27,40}$ The high quantum yield of $1 \mathrm{a}$ implies that this compound is almost completely present in acetonitrile solutions as antiparallel conformer, whose photoreaction is very efficient. High ring-closure quantum yields are also shown by diarylethenes having $\pi$-donor substituents on the thiophene rings, ${ }^{21,28,39}$ and for some of these compounds a prevalence of the antiparallel conformer has been detected. ${ }^{28}$ It has also been suggested that polar solvents favor antiparallel conformers, ${ }^{6}$ but inconsistent results have been obtained about the effect of the solvent on the ring-closure quantum yield. ${ }^{33,40,41}$

The ring-closure quantum yield of $\mathbf{1 a}$ is practically constant when irradiation is performed in the lowest-energy absorption band (313 or $334 \mathrm{~nm}, \Phi=0.7)$, but a higher $(\Phi=0.9)$ upon excitation in the second band $(254 \mathrm{~nm})$; no explanation can be advanced for this difference.

Ring-opening photoreaction. Considering the closed isomer 1b, it exhibits a very low ring-opening quantum yield (see Table 2), as occurs for many other diarylethenes. ${ }^{21-23,28,40,42,43}$ The large difference between ring-closure and ring-opening quantum yields may find an explanation in the theoretical calculations of the ground- and excited-states potential energy surfaces along the reaction coordinate: the reaction path of open isomers is practically barrierless, while an activation energy barrier appears in that of closed isomers. ${ }^{23,27,29,31,33,44}$ As a consequence, ring-closure photoreaction is the main process originated by excited states of open isomers, while the most efficient process originated by excited states of closed isomers is the deactivation to their ground state. The presence of an energy barrier can also explain why the ringopening quantum yield increases with the energy of the irradiation light (see Fig. 5).

Secondary photoreaction and fatigue resistance. Many diarylethene compounds have been found to undergo secondary photoreactions to stable products, besides the main ring-closure and ring-opening photoreactions. ${ }^{20,24,25,42,44,45}$ In the case of 1,2-bis(5-phenyl-2-methylthiophen-3-yl)perfluorocyclopentene ${ }^{42}$ and 1,2-bis(2,5-dimethylthiophen-3-yl)perfluorocyclopentene ${ }^{25}$ the major secondary product (in deaerated hexane solutions) has been isolated and characterized: it is a colored isomeric form of the reactant in which the thiophene rings have formed a condensed system with two six-membered heterocyclic rings. These compounds showed up in the visible absorption spectra similarly to the closed-ring forms but with less intensity (about $40 \%$ ) and slightly shifted to shorter wavelengths; the similar characteristics of the small increase of absorbance we have observed around $560 \mathrm{~nm}$ lead to the assumption that the secondary product formed by UV irradiation of $\mathbf{1 b}$ is of the same nature. It is important to note that the secondary products seem 
to be formed by UV irradiation of closed isomers but they are not obtained upon irradiation with visible light, ${ }^{25,42}$ which means that the side reactions only originate from high-energy excited states.

The fatigue resistance of $\mathbf{1}$ is comparable to that of analogous compounds. ${ }^{4}$ The decrease of the fatigue resistance observed in our experiments in aerated solutions (Table 3 ) is a rather common effect, ${ }^{4,42}$ caused by the formation of oxygenated products, possibly endo-peroxide moieties on the thiophene rings. ${ }^{46}$ Because of the reasons stated above, the secondary photoreactions with $\mathrm{O}_{2}$ must originate from triplet excited states.

\section{Compound 1 as actinometer}

The reversible photocyclization reaction which compound $\mathbf{1}$ undergoes when irradiated with UV or visible light offers the possibility of using it as an easy chemical actinometer. A photochromic actinometer is very convenient and its use very simple. Indeed, (i) a spectrophotometric cell containing a solution of the compound can be utilized for a large number of actinometric measurements; (ii) the analytical determination of the photolyzed molecules is rapid, consisting of only a few spectrophotometric measurements before and after irradiation. Furthermore, compound 1 covers a wide spectral range, both in the UV (250$340 \mathrm{~nm}$ ), where other actinometers are also available, and, albeit with small quantum yield values, in the visible $(400-620 \mathrm{~nm})$, where only a few actinometers can be used, but with severe limitations. As for other actinometers, it is necessary to take the precaution of avoiding biphotonic processes when used for measuring the light intensity emitted by very powerful lasers, since they may be much more efficient than the monophotonic processes. ${ }^{43,47}$

\section{Comparison with the most common actinometers}

The photochromic compound $\mathbf{1}$ presents some advantages over traditional actinometers; for comparison purposes, here we will consider only the most commonly used, all characterized by simple spectrophotometric measurements in the analytical method.

Ferrioxalate. ${ }^{14,15}$ Based on the photolytic decomposition of potassium ferrioxalate, it is one of the most popular actinometers, that can be easily used in the range $250-465 \mathrm{~nm}$, and with more difficulty and less accuracy up to $550 \mathrm{~nm}$, because beyond $465 \mathrm{~nm}$ the procedure requires large corrections, due to the small molar extinction coefficients. The dependence of the quantum yield on the irradiation wavelength was extensively studied. ${ }^{13}$ The analytical procedure implies only the addition of a reactant to the irradiated solution, although a comparison with a solution kept in the dark at the same temperature is required.

Reinecke's salt. ${ }^{16}$ Another actinometric compound, which can be used conveniently from 315 up to $600 \mathrm{~nm}$, is Reinecke's complex $\mathrm{K}\left[\mathrm{Cr}\left(\mathrm{NH}_{3}\right)_{2}(\mathrm{SCN})_{4}\right]$, which upon irradiation undergoes a photoaquation reaction with release of a $\mathrm{SCN}^{-}$ion. ${ }^{16}$ The quantum yield of the photoreaction shows only a slight dependence on the irradiation wavelength, but the practical procedure is not simple: the $\mathrm{SCN}^{-}$ions are also formed by a fast thermal reaction, so that the solution has to be prepared immediately before the use; also with this precaution the thermal reaction causes troubles in the analytical procedure. ${ }^{13}$
Aberchrome 540. In the past, the use of a fulgide, $(E)-2-[1-(2,5-$ dimethyl-3-furylethylidene]-3-isopropylidene succinic anhydride, was also suggested. ${ }^{17}$ The commercial trade name was Aberchrome 540 , but nowadays it is no longer commercially available, although easy to prepare. This is a photochromic compound that, when irradiated in the open form with UV light (320-370 nm) undergoes a photocyclization reaction, giving a coloured compound, thermally stable in the experimental conditions used; ${ }^{17-19}$ this closed form gives back the open one upon irradiation with visible light (435-545 nm). A very simple practical procedure is required, so this actinometer is very convenient. However, further studies have evidenced that the open form in the UV region also undergoes a secondary undesired photoisomerization reaction $(E \rightleftharpoons Z) .{ }^{13}$ As both $E$ and $Z$ isomers of the open form do not absorb visible light, the repeated use of this actinometer is therefore recommended only in the visible range. ${ }^{13}$

Compound $\mathbf{1}$ is a very simple and reusable photochromic actinometer, which exists in two thermally stable isomeric forms, 1a (open-ring isomer) exploitable from 254 up to $340 \mathrm{~nm}$, while 1b (closed-ring isomer) can be utilized in the visible region (400$620 \mathrm{~nm}$ ), a spectral range where 1 shows many advantages in respect to the few available actinometers. Furthermore, compound $\mathbf{1}$ is a very fatigue resistant compound, a property which allows the use of the same solution for a large number of actinometric measurements. Fig. 7 compares the spectral ranges of availability for the above mentioned actinometers with that of compound $\mathbf{1}$. Practical indications for the use of compound $\mathbf{1}$ as actinometer are listed in the Appendix.

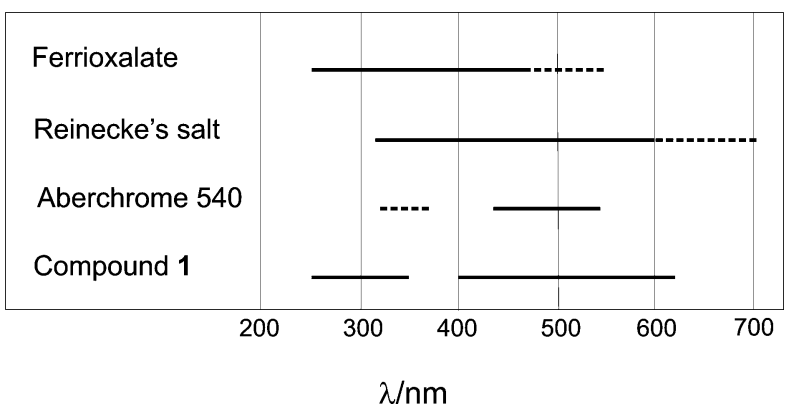

Fig. 7 Spectral range of availability of the most common actinometers in comparison with compound $\mathbf{1}$. The dashed lines indicate the range of wavelengths where the use is possible, but not recommended or simple.

\section{Appendix}

\section{Recommended practical actinometric procedure}

An approximately $1 \times 10^{-4} \mathrm{~mol} \mathrm{~L}^{-1}$ solution of $\mathbf{1}$ is prepared in acetonitrile (Merck Uvasol or equivalent) and a known volume of this solution is put in a sealable quartz spectrophotometric cell, containing a magnetic bar to stir the solution during irradiation; take care that the magnetic bar does not interfere with the light beam. Deoxygenation of the solution is desired to improve the fatigue resistance of the system. The actinometric solution must be stored in the dark, preferably in the $\mathbf{1 a}$ form.

UV region. Read the absorbance at both the wavelength of excitation and $600 \mathrm{~nm}$, before and after irradiation. The increase 
in absorbance at $600 \mathrm{~nm}(0.1-0.2)$ enables the photon intensity to be calculated using eqn (4),

$$
I_{\mathrm{o}}=\frac{\Delta A_{600} \times V}{\Phi_{\lambda}(\mathbf{1} \mathbf{a} \rightarrow \mathbf{1 b}) \times\left(1-10^{-A \lambda}\right) \times f_{\lambda}(\mathbf{1} \mathbf{a}) \times \varepsilon_{600} \times d \times \Delta t}
$$

where:

$\Delta A_{600}$ is the increase of absorbance at $600 \mathrm{~nm}$ in the irradiation period $\Delta t$;

$V$ is the volume of irradiated solution (L);

$\Phi_{\lambda}(\mathbf{1 a} \rightarrow \mathbf{1 b})$ is the ring-closure quantum yield at the excitation wavelength;

$A_{\lambda}$ is the absorbance of the solution at the excitation wavelength (mean value during the irradiation period);

$\varepsilon_{600}$ is the molar absorption coefficient of $1 \mathbf{b}$ at $600 \mathrm{~nm}(17400 \mathrm{~L}$ $\left.\mathrm{mol}^{-1} \mathrm{~cm}^{-1}\right)$;

$d$ is the optical path of the actinometric cell $(\mathrm{cm})$;

$\Delta t$ is the irradiation time ( $\mathrm{min})$;

$f_{\lambda}$ (1a) represents the fraction of the total absorbed light that is actually absorbed by $\mathbf{1 a}$ and it can be calculated by means of eqn (5),

$$
f_{\lambda}(\mathbf{1 a})=1-\frac{C_{\mathrm{m}} \times \varepsilon_{\lambda} \times d}{A_{\lambda}}
$$

where $C_{\mathrm{m}}$ is the mean molar concentration of $\mathbf{1 b}$ in the irradiation period (obtained from the absorbance values at $600 \mathrm{~nm}$ ) and $\varepsilon_{\lambda}$ is the molar absorption coefficient of $\mathbf{1 b}$ at the excitation wavelength.

Note that, if no more that $10 \%$ of $\mathbf{1 a}$ is converted to $\mathbf{1 b}$, in the spectral range $250-340 \mathrm{~nm}, f_{\lambda}(\mathbf{1 a})$ is larger than 0.94 ; moreover, for $d \geq 1 \mathrm{~cm}$, the excitation light is completely absorbed by the solution $(98.5 \%$ at $254 \mathrm{~nm})$, so that the term $\left(1-10^{-A} \lambda\right)$ can be neglected in eqn (4). For $\lambda>340 \mathrm{~nm}$, the absorption coefficient of 1b is larger than that of $\mathbf{1 a}$ and so the fraction of light absorbed by $\mathbf{1 b}$ is high even for small conversions (e.g. at $365 \mathrm{~nm} \mathbf{1 b}$ absorbs $59 \%$ of the light after $10 \%$ conversion). The use of 1 as actinometer in the 340-400 $\mathrm{nm}$ spectral region is, therefore, discouraged.

Visible region. To prepare a suitable actinometric solution, the starting solution of 1a is irradiated with UV light until the absorbance at $600 \mathrm{~nm}$ reaches a sufficiently high value (1.0-1.5). It is convenient to use light close to the absorption maximum of 1a $(313 \mathrm{~nm})$ in order to minimize the light absorption by $\mathbf{1 b}$ and increase the fatigue resistance. A final value of 1.0 for the absorbance at $600 \mathrm{~nm}$ (corresponding to $[\mathbf{1 b}]=5.8 \times 10^{-5} \mathrm{~mol} \mathrm{~L}^{-1}$ ) is a convenient compromise between a high absorption of visible light by the actinometric solution $(90 \%$ at $600 \mathrm{~nm})$ and the fraction of light absorbed by $\mathbf{1 b}$ in the UV; we recommend reaching the 1.5 value only for actinometric measurements around the minimum of $\mathbf{1 b}$ spectrum $(430 \mathrm{~nm})$. Now the solution is ready for irradiation at the desired visible wavelength: read the absorbance at $600 \mathrm{~nm}$ and at the excitation wavelength and measure again these values after a suitable irradiation period; the decrease in absorbance at $600 \mathrm{~nm}$ can now be used to calculate the light intensity using eqn (6),

$$
I_{\mathrm{o}}=\frac{\Delta A_{600} \times V}{\Phi_{\lambda}(\mathbf{1} \mathbf{b} \rightarrow \mathbf{1 a}) \times\left(1-10^{-A \lambda}\right) \times \varepsilon_{600} \times d \times \Delta t}
$$

where:

$\Delta A_{600}$ is now the decrease of absorbance at $600 \mathrm{~nm}$ in the irradiation period $\Delta t$;
$\Phi_{\lambda}(\mathbf{1 b} \rightarrow \mathbf{1 a})$ is the ring-opening quantum yield at the excitation wavelength, which can be derived from eqn (3);

the other symbols have the same meaning as for eqn (4).

A term like $f_{\lambda}(\mathbf{1 a})$ of eqn (4) is not required in this case since 1a does not absorb in the visible.

Fatigue resistance. We have calculated that, working in the conditions stated above and assuming a quantum yield for the formation of secondary products $\Phi_{\mathrm{sp}}=0.001$ (see Results, Photochemical behaviour), the amount of secondary products produced during a single measurement should be less than $5 \times$ $10^{-10} \mathrm{~mol} \mathrm{~L}^{-1}$ for UV-light and less than $8 \times 10^{-8} \mathrm{~mol} \mathrm{~L}^{-1}$ for visiblelight intensity evaluation, respectively. Therefore one actinometric solution can be used about 500 times with less than $10 \%$ loss of compound $\mathbf{1 .}$

\section{References}

$1 \mathrm{H}$. Bouas-Laurent and H. Dürr, Organic photochemistry (IUPAC Technical Report), Pure Appl. Chem., 2001, 73, 639-665.

2 S. E. Braslavsky, Glossary of terms used in photochemistry, 3rd edition (IUPAC Recommendations 2006), Pure Appl. Chem., 2007, 79, 293465.

3 M. Irie, Photochromic diarylethenes for photonic devices, Pure Appl. Chem., 1996, 68, 1367-1371.

4 M. Irie, Diarylethenes for memories and switches, Chem. Rev., 2000, 100, 1685-1716, and references therein.

$5 \mathrm{~K}$. Uchida, Y. Nakayama and M. Irie, Thermally irreversible photochromic systems. Reversible photocyclization of 1,2-bis(benzo[b]thiophen-3-yl)ethene derivatives, Bull. Chem. Soc. Jpn., 1990, 63, 13111315.

6 A. Goldberg, A. Murakami, K. Kanda, T. Kobayashi, S. Nakamura, K. Uchida, H. Sekiya, T. Fukaminato, T. Kawai, S. Kobatake and M. Irie, Rotational isomerization of dithienylethenes: a study on the mechanism determining quantum yield of cyclization reaction, J. Phys. Chem. A, 2003, 107, 4982-4988.

7 R. B. Woodward and R. Hoffmann, The Conservation of Orbital Symmetry, Verlag Chemie, GmbH, Weinheim, 1970.

8 S. Nakamura and M. Irie, Thermally irreversible photochromic systems. A theoretical study, J. Org. Chem., 1988, 53, 6136-6138.

9 A. Osuka, D. Fujikane, H. Shinmori, S. Kobatake and M. Irie, Synthesis and photoisomerization of dithienylethene-bridged diporphyrins, J. Org. Chem., 2001, 66, 3913-3923.

10 V. M. Iyer, H. Stoeckli-Evans and P. Belser, A perfluorocyclopentene dithienylethene based molecular switch: 1,2-bis[5-(4-ethynylphenyl)-2methylthiophen-3yl]-3,3,4,4,5,5-hexafluorocyclopentene, Acta Crystallogr., Sect. E: Struct. Rep. Online, 2006, 62, o3942-o3943.

11 The spot of the colorless 1a isomer was obviously not visible in the plate, but it turned colored upon irradiation with $254 \mathrm{~nm}$ light, revealing a solid state photoconversion.

12 H. J. Kuhn, S. E. Braslavsky and R. Schmidt, Chemical actinometry (IUPAC Technical Report), Pure Appl. Chem., 2004, 76, 2105-2146.

13 M. Montalti, A. Credi, L. Prodi and M. T. Gandolfi, Handbook of Photochemistry, CRC press, Boca Raton, FL, USA, 3rd edn, 2006, pp. 583-561.

14 C. G. Hatchard and C. A. Parker, A new sensitive chemical actinometer. II. Potassium, ferric oxalate as a standard chemical actinometer, Proc. R. Soc. London, Ser. A, 1956, 235, 518-536.

15 E. Fisher, Ferri-oxalate actinometry, EPA Newsletters, 1984, 21, 33-34.

16 E. E. Wegner and A. W. Adamson, Photochemistry of complex ions. III. Absolute quantum yields for the photolysis of some aqueous chromium(III) complexes. Chemical actinometry in the long wavelength visible region, J. Am. Chem. Soc., 1966, 88, 394- 404.

$17 \mathrm{H}$. G. Heller and J. R. Langan, Photochromic heterocyclic fulgides. Part 3. The use of (E)-a-(2,5-dimethyl-3-furylethylidene)(isopropylidene)succinic anhydride as a simple convenient chemical actinometer, J. Chem. Soc., Perkin Trans. 2, 1981, 341-343.

18 P. J. Darcy, H. G. Heller, P. J. Strydom and J. Wittal, Photochromic heterocyclic fulgides. Part 2. Electrocyclic reactions of $(E)$-a-2,5dimethyl-3-furylethylidene(alkyl-substituted methylene) succinic anhydrides, J. Chem. Soc., Perkin Trans. 1, 1981, 202-205. 
19 A. P. Glaze, H. G. Heller and J. Wittal, Photochromic heterocyclic fulgides. Part 7. (E)-adamantylidene-[1-(2,5-dimethyl-3furyl)ethylidene]succinic anhydride and derivatives: model photochromic compounds for optical recording media, J. Chem. Soc., Perkin Trans. 2, 1992, 591-594.

20 S. L. Gilat, S. H. Kawai and J.-M. Lehn, Light-triggered molecular devices: photochemical switching of optical and electrochemical properties in molecular wire type diarylethene species, Chem.-Eur. J., 1995, $1,275-284$

21 M. Irie, K. Sakemura, M. Okinaka and K. Uchida, Photochromism of dithienylethenes with electron-donating substituents, J. Org. Chem., 1995, 60, 8305-8309.

22 M. Irie, T. Eriguchi, T. Takada and K. Uchida, Photochromism of diarylethenes having thiophene oligomers as the aryl groups, Tetrahedron, 1997, 53, 12263-12271.

23 M. Irie, T. Lifka, S. Kobatake and N. Kato, Photochromism of 1,2-bis(2-methyl-5-phenyl-3-thienyl)perfluorocyclopentene in a singlecrystalline phase, J. Am. Chem. Soc., 2000, 122, 4871-4876.

24 J. J. D. de Jong, L. N. Lucas, R. Hania, A. Pugzlys, R. M. Kellogg, B. L. Feringa, K. Duppen and J. H. van Esch, Photochromic properties of perhydro- and perfluorodithienylcyclopentene molecular switches, Eur. J. Org. Chem., 2003, 1887-1893.

25 K. Higashiguchi, K. Matsuda, S. Kobatake, T. Yamada, T. Kawai and M. Irie, Fatigue mechanism of photochromic 1,2-bis(2,5-dimethyl-3thienyl)perfluorocyclopentene, Bull. Chem. Soc. Jpn., 2000, 73, 23892394.

26 The correction can also be accomplished mathematically, dividing the quantum yield values obtained with eqn (2) by $\left[1-\left(C_{\mathrm{m}} \times \varepsilon_{\lambda} / A_{\lambda}\right)\right]$; $C_{\mathrm{m}}$ is the mean molar concentration of $\mathbf{1 b}$ in the irradiation period (obtained from the absorbance values at $600 \mathrm{~nm}$ ), $\varepsilon_{\lambda}$ and $A_{\lambda}$ are the extinction coefficient of $\mathbf{1 b}$ and the mean absorbance of the solution at the irradiation wavelength, respectively. This correction factor represents the fraction of the absorbed light which is actually absorbed by $\mathbf{1 a}$.

27 M. Irie and M. Mohri, Thermally irreversible photochromic systems. Reversible photocyclization of diarylethene derivatives, J. Org. Chem., 1988, 53, 803-808.

28 J. Ern, A. T. Bens, H.-D. Martin, K. Kuldova, H. P. Trommsdorff and C. Kryschi, Ring-opening and-closure reaction dynamics of a photochromic dithienylethene derivative, J. Phys. Chem. A, 2002, 106, 1654-1660.

29 J. Ern, A. T. Bens, H.-D. Martin, S. Mukamel, D. Schmid, S. Tretiak, E. Tsiper and C. Kryschi, Reaction dynamics of photochromic dithienylethene derivatives, Chem. Phys., 1999, 246, 115-125.

30 P. R. Hania, R. Telesca, L. N. Lucas, A. Pugzlys, J. van Esch, B. L. Feringa, J. G. Snijders and K. Duppen, An optical and theoretical investigation on the ultrafast dynamics of a bisthienylethene-based photochromic switch, J. Phys. Chem. A, 2002, 106, 8498-8507.

31 P. R. Hania, A. Pugzlys, L. N. Lucas, J. J. D. de Jong, B. L. Feringa, J. H. van Esch, H. T. Jonkman and K. Duppen, Ring closure dynamics of BTE-based photochromic switches: perfluoroversus perhydrocyclopentene derivatives, J. Phys. Chem. A, 2005, 109, 9437-9442.

32 A. T. Bens, D. Frewert, K. Kodatis, C. Kryschi, H.-D. Martin and H. P. Trommsdorff, Coupling of chromophores: carotenoids and photoactive diarylethenes - Photoreactivity versus radiationless deactivation, Eur. J. Org. Chem., 1998, 2333-2338.

33 J. Ern, A. T. Bens, H.-D. Martin, S. Mukamel, S. Tretiak, K. Tsyganenko, K. Kuldova, H. P. Trommsdorff and C. Kryschi, Reaction dynamics of a photochromic fluorescing dithienylethene, J. Phys. Chem. A, 2001, 105, 1741-1749.

$34 \mathrm{~N}$. Tamai and H. Miyasaka, Ultrafast dynamics of photochromis systems, Chem. Rev., 2000, 100, 1875-1890, and references therein.

35 J. C. Owrutsky, H. H. Nelson, A. P. Baronavski, O.-K. Kim, G. M. Tsivgoulis, S. L. Gilat and J.-M. Lehn, Optical properties and dynamics of a photochromic bisthienylethene in solution and in a polymer film, Chem. Phys. Lett., 1998, 293, 555-563.

36 C. Okabe, T. Nakabayashi, N. Nishi, T. Fukaminato, T. Kawai, M. Irie and H. Sekiya, Picosecond time-resolved Stokes and antiStokes Raman studies on the photochromic reactions of diarylethene derivatives, J. Phys. Chem. A, 2003, 107, 5384-5390.

37 S. Shim, T. Joo, S. Chul Bae, K. S. Kim and E. Kim, Ring opening dynamics of a photochromic diarylethene derivative in solution, J. Phys. Chem. A, 2003, 107, 8106-8110.

38 Ref. 13 , pp. $421-424$.

39 R. T. F. Jukes, V. Adamo, F. Harti, P. Belser and L. De Cola, Photochromic dithienylethene derivatives containing Ru(II) or Os(II) metal units. Sensitized Photocyclization from a triplet state, Inorg. Chem., 2004, 43, 2779-2792.

$40 \mathrm{M}$. Irie and K. Sayo, Solvent effects on the photochromic reactions of diarylethene derivatives, J. Phys. Chem., 1992, 96, 76717674.

41 H. Miyasaka, T. Nobuto, M. Murakami, A. Itaya, N. Tamai and M. Irie, Solvent viscosity effects on photochromic reactions of a diarylethene derivative as revealed by picosecond laser spectroscopy, J. Phys. Chem. A, 2002, 106, 8096-8102.

42 M. Irie, T. Lifka, K. Uchida, S. Kobatake and Y. Shindo, Fatigue resistant properties of photochromic diarylethenes: by-product formation, Chem. Commun., 1999, 747-750.

43 M. Murakami, H. Miyasaka, T. Okada, S. Kobatake and M. Irie, Dynamics and mechanisms of the multiphoton gated photochromic reaction of diarylethene derivatives, J. Am. Chem. Soc., 2004, 126, 14764-14772.

44 L. N. Lucas, J. J. D. de Jong, J. H. van Esch, R. M. Kellogg and B. L. Feringa, Syntheses of dithienylcyclopentene optical molecular switches, Eur. J. Org. Chem., 2003, 155-166.

45 M. Hanazawa, R. Sumiya, Y. Horikawa and M. Irie, Thermally irreversible photochromic systems. Reversible photocyclization of 1,2-bis(2-methylbenzo[b]thiophen-3-yl)perfluorocycloalkene derivatives, J. Chem. Soc., Chem. Commun., 1992, 206-207.

46 H. Taniguchi, A. Shinpo, T. Okazaki, F. Matsui and M. Irie, Photodegradation mechanism of photochromic diarylethene derivatives, Nippon Kagaku Kaishi, 1992, 1138-1140 (Chem. Abs., 1993, 118, 59154 $\mathrm{m})$.

47 M. Miyasaka, M. Murakami, T. Okada, Y. Nagata, A. Itaya, S. Kobatake and M. Irie, Picosecond and femtosecond laser photolysis studies of a photochromic diarylethene derivative: multiphoton gated reaction, Chem. Phys. Lett., 2003, 371, 40-48. 